\title{
Initial medical therapy for moderate to severe ISCHEMIA
}

Taking time
to optimize
medical
therapy
and assess
response to
therapy does
not expose
patients to
greater risk

The addition of an invasive strategy (angiography plus revascularization when feasible) to medical therapy does not reduce the risk of cardiovascular events or death but does reduce symptoms of angina among patients with stable coronary artery disease (CAD) and moderate or severe ischaemia. These findings from the ISCHEMIA trial have now been published in NEJM.

Patients with stable CAD, also known as chronic coronary syndrome, can be treated conservatively with the use of medical therapy (antianginal drugs plus diseasemodifying drugs such as lipidlowering, antithrombotic and renin-angiotensin-blocking agents) with or without an invasive strategy involving coronary angiography followed by percutaneous coronary intervention or coronary artery bypass graft surgery. Clinical equipoise exists as to the optimal

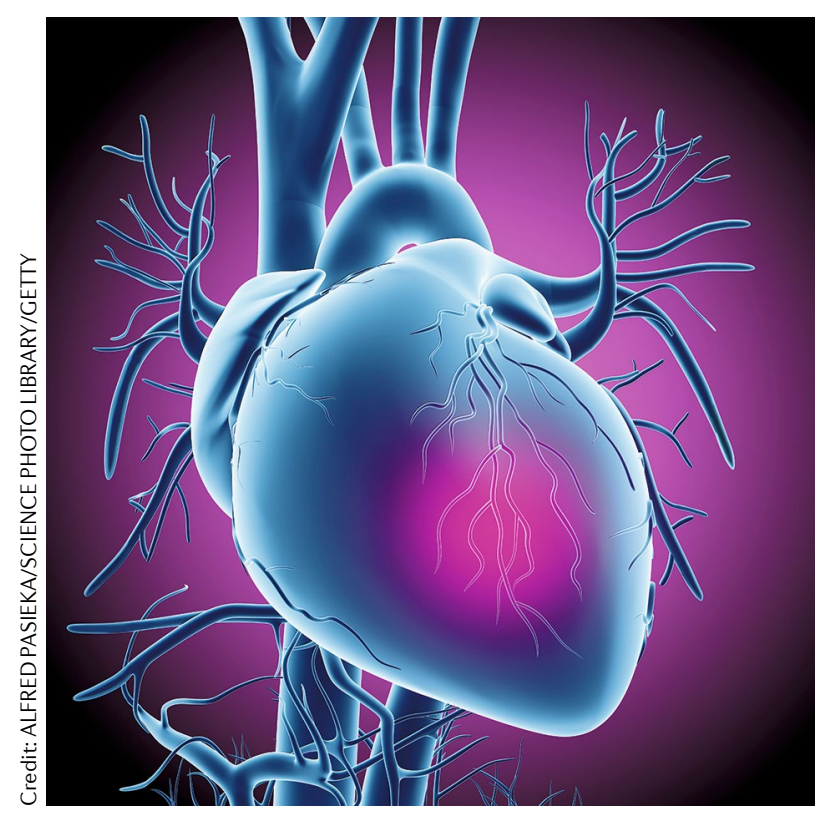

approach, leading David Maron, Judith Hochman and colleagues to conduct the ISCHEMIA trial.

A total of 5,179 patients with stable CAD and moderate or severe ischaemia were randomly assigned to an initial invasive strategy plus medical therapy or an initial strategy of medical therapy alone followed by angiography if medical therapy was unsuccessful.

During follow-up (median 3.2 years), a primary outcome event (death from cardiovascular causes, myocardial infarction, or hospitalization for unstable angina, heart failure or resuscitated cardiac arrest) occurred in 318 patients in the invasive-strategy group and 352 patients in the conservativestrategy group. At 5 years, the cumulative event rate was not significantly different between the two groups (16.4\% versus $18.2 \%$ ). Similarly, the risk of death did not differ significantly according to treatment strategy.

Of note, the definition of myocardial infarction (whether or not procedural infarctions were included) had an effect on the analysis. The invasive strategy was associated with a higher rate of procedural myocardial infarctions within the first 6 months, whereas the conservative strategy was associated with more (predominantly spontaneous) myocardial infarctions as the trial continued. "Although there was no difference in mortality between the groups," comments Maron, "it will be important to follow up for the longer term to determine if a survival benefit emerges for the invasive approach."

In a second paper published in NEJM, the ISCHEMIA investigators report assessments of angina-related symptoms, function and quality of life with the Seattle Angina Questionnaire. Patients assigned to the invasive strategy had greater improvements in angina-related health status than those assigned to the conservative strategy. The benefit was confined to the $65 \%$ of patients who had angina in the month before randomization.

In another paper published in JAMA Cardiology, the ISCHEMIA investigators report a secondary analysis into sex-specific differences. Women, who comprised $23 \%$ of the trial population, had a higher frequency of angina than men, despite having less severe ischaemia on stress testing and less extensive CAD on coronary CT angiography. The investigators attribute these findings to inherent sex-specific differences in the complex relationships between angina, atherosclerosis and ischaemia.

"ISCHEMIA confirms that there is no need to rush to the catheterization laboratory after learning of an abnormal stress test," summarizes Maron. "Taking time to optimize medical therapy and assess response to therapy does not expose patients to greater risk," he adds. Therefore, an initial conservative strategy should be recommended for patients who fit the profile of those included in the ISCHEMIA trial. An invasive approach is appropriate for those with angina whose symptoms are not adequately relieved with medical therapy.

Gregory B. Lim

ORIGINAL ARTICLES Maron, D. J. et al. Initial
invasive or conservative strategy for stable
coronary disease. N. Engl. J. Med. https://doi.org/
10.1056/NEJMoa1915922 (2020) | Spertus, J. A. et al.
Health-status outcomes with invasive or
conservative care in coronary disease. N. Engl.
J. Med. https://doi.org/10.1056/NEJMoa1916370
(2020) | Reynolds, H. R. et al. Association of sex
with severity of coronary artery disease, ischemia,
and symptom burden in patients with moderate
or severe ischemia: secondary analysis of the
ISCHEMIA randomized clinical trial.JAMA Cardiol.
https://doi.org/10.1001/jamacardio.2020.0822
(2020)

ORIGINAL ARTICLES Maron, D. J. et al. Initial invasive or conservative strategy for stable coronary disease. N. Engl.J. Med. https://doi.org/ 10.1056/NEJMoa1915922 (2020) |Spertus, J. A. et al. Health-status outcomes with invasive or conservative care in coronary disease. N. Engl. . (2020) | Reynolds, H. R. et al. Association of sex with severity of coronary artery disease, ischemia, or severe ischemia: secondary analysis of the ISCHEMIA randomized clinical trial.JAMA Cardio. (2020) 